\title{
Randomized phase II study of paclitaxel/ carboplatin intercalated with gefitinib compared to paclitaxel/carboplatin alone for chemotherapy- naïve non-small cell lung cancer in a clinically selected population excluding patients with non- smoking adenocarcinoma or mutated EGFR
}

Yoon Ji Choi ${ }^{1,2}$, Dae Ho Lee ${ }^{1,3,4}$, Chang Min Choi ${ }^{1,3,4}$, Jung Shin Lee ${ }^{1,3,4}$, Seung Jin Lee ${ }^{3}$, Jin-Hee Ahn 1,3,4 and Sang-We Kim ${ }^{1,3,4^{*}}$

\begin{abstract}
Background: Considering cell cycle dependent cytotoxicity, intercalation of chemotherapy and epidermal growth factor receptor (EGFR) tyrosine kinase inhibitor (TKI) may be a treatment option in non-small cell lung cancer (NSCLC). This randomized phase 2 study compared the efficacy of paclitaxel and carboplatin (PC) intercalated with gefitinib $(G)$ versus $P C$ alone in a selected, chemotherapy-naïve population of advanced NSCLC patients with a history of smoking or wild-type EGFR.
\end{abstract}

Methods: Eligible patients were chemotherapy-naïve advanced NSCLC patients with Eastern Cooperative Oncology Group performance status of 0 -2. Non-smoking patients with adenocarcinoma or patients with activating EGFR mutation were excluded because they could benefit from gefitinib alone. Eligible patients were randomized to one of the following treatment arms: PCG, P $175 \mathrm{mg} / \mathrm{m}^{2}$, and C AUC 5 administered intravenously on day 1 intercalated with G 250 mg orally on days 2 through 15 every 3 weeks for four cycles followed by G 250 mg orally until progressive disease; or PC, same dosing schedule for four cycles only. The primary endpoint was the objective response rate (ORR), and the secondary endpoints included progression-free survival (PFS), overall survival (OS), and toxicity profile.

Results: A total of 90 patients participated in the study. The ORRs were $41.9 \%$ (95\% confidence interval (Cl) 27.0$57.9 \%)$ for the PCG arm and $39.5 \%$ (95\% Cl 25.0-55.6\%) for the PC arm ( $P=0.826)$. No differences in PFS (4.1 vs. 4.1 months, $P=0.781$ ) or OS ( 9.3 vs. 10.5 months, $P=0.827$ ) were observed between the PCG and PC arms. Safety analyses showed a similar incidence of drug-related grade $3 / 4$ toxicity. Rash and pruritus were more frequent in the PCG than in the PC arm.

(Continued on next page)

\footnotetext{
* Correspondence: swkim@amc.seoul.kr

Presented in part at the 2013 AACR-NCI-EORTC International Conference on Molecular Targets and Cancer Therapeutics October19th-23th, 2013, Boston MA, USA.

'Department of Oncology, Asan Medical Center, University of Ulsan College of Medicine, 88 Olympic-ro-43-gil, Songpa-gu, Seoul 138-736, Korea

${ }^{3}$ Asan Institute for Life Science, Asan Medical Center, Institute for Innovative Cancer Research, Seoul, Korea

Full list of author information is available at the end of the article
} 
(Continued from previous page)

Conclusions: PCG did not improve ORR, PFS, and OS compared to PC chemotherapy alone for NSCLC in a clinically selected population excluding non-smoking adenocarcinoma or mutated EGFR.

Trial registration: The study is registered with ClinicalTrials.gov (NCT01196234). Registration date is 08/09/2010.

Keywords: Non-small cell lung cancer, Intercalated chemotherapy, Gefitinib, Smoker, Wild-Type EGFR

\section{Background}

Despite significant advances in the treatment of nonsmall-cell lung cancer (NSCLC) over the last two decades, the results of standard chemotherapy for advanced NSCLC have reached a plateau and new treatment strategies are necessary. The introduction of epidermal growth factor receptor (EGFR) inhibitors was considered a promising strategy. EGFR-tyrosine-kinase inhibitors (TKIs), specifically gefitinib and erlotinib, are currently considered the standard first-line treatment for patients with activating EGFR mutations based on the results of several randomized studies [1-6]. However, the benefit of these agents is confined to patients with EGFR mutation [7, 8].

The mechanism of action of EGFR inhibitors is the inhibition of tumor cell proliferation and induction of apoptosis. The addition of EGFR inhibitors to standard chemotherapy is an attractive approach to enhance its efficacy. However, no survival advantage was detected in trials such as the INTACT I, II, and TRIBUTE studies [9-11]. One possible explanation for the failure of these studies is that tumor cells that were driven to G0/G1 phase by EGFR TKIs may not be sensitive to cytotoxic chemotherapy. Preclinical studies and several phase 1 and 2 studies showed that sequential treatment with chemotherapy followed by EGFR TKIs led to synergistic cytotoxicity [12-16].

The present study examined the effect of gefitinib administered for 2 weeks after paclitaxel and carboplatin (PC) chemotherapy by assessing cell-cycle progression during chemotherapy in patients with NSCLC. Nonsmoking patients with adenocarcinoma and patients with mutant EGFR, who were expected to benefit from gefitinib alone, were excluded from the analysis.

\section{Methods}

\section{Study design and population}

This study was a single-center, prospective, open-label, randomized phase II study of paclitaxel and carboplatin with intercalated gefitinib (PCG) or PC alone (PC) for advanced NSCLC in a selected population of smokers with wild-type EGFR.

Patients were eligible for this study if they were 18 years or older, had a histological diagnosis of NSCLC with metastasis (stage IV) or locally advanced (stage IIIB) disease with malignant pleural effusion according to the 6th edition of the American Joint Committee on
Cancer staging system. Inclusion criteria were $\geq 1$ measurable lesion meeting Response Evaluation Criteria in Solid Tumors (RECIST version 1.1) guidelines, an Eastern Cooperative Oncology Group (ECOG) performance status (PS) of $0-2$, at least 1 week since the last radiotherapy session, and adequate organ function.

Exclusion criteria included tumors harboring EGFR mutation, prior systemic chemotherapy for NSCLC, non-smoking patients with adenocarcinoma (except patients with wild-type EGFR), symptomatic brain metastasis and any unstable medical condition.

This study was approved by the institutional review board of Asan Medical Center and was performed in accordance with the Declaration of Helsinki and Good Clinical Practice guidelines. All patients gave written informed consent before treatment. This study has been submitted for registration with ClinicalTrials.gov identifier NCT01196234.

\section{Randomization}

Eligible patients were randomly assigned in a 1:1 ratio to receive PCG or PC alone. Randomization was stratified by gender and histology (adenocarcinoma, others).

\section{Treatment plan}

Patients in the PCG arm received paclitaxel $175 \mathrm{mg} / \mathrm{m}^{2}$ and carboplatin AUC 5 intravenously on day 1 with intercalated gefitinib $250 \mathrm{mg}$ orally once daily from day 2 through day 15 every 3 weeks for four cycles followed by gefitinib $250 \mathrm{mg}$ orally until progressive disease or unacceptable toxicity. Patients in the PC arm received paclitaxel $175 \mathrm{mg} / \mathrm{m}^{2}$ and carboplatin AUC 5 intravenously on day 1 every 3 weeks until progressive disease up to four cycles without following maintenance therapy.

Dose adjustments were based on drug-related toxicities. When dose reduction was necessary, gefitinib was stopped every third day. If a patient still required dose reduction, it was stopped every other day. Any patient who required three dose reductions or developed interstitial lung disease (ILD) was discontinued from the study drug. Treatment could be delayed for a maximum of 2 weeks.

\section{Evaluation}

Physical examination, ECOG PS evaluation and toxicity rating according to the Common Terminology Criteria 
for Adverse Events (CTCAE version 3.0) were performed at baseline, prior to each cycle, and approximately 1 month after the last dose of the study drug. Tumor response was assessed by computed tomography (CT) with RECIST version 1.1 every 6 weeks during chemotherapy, and every 12 weeks thereafter until disease progression.

\section{Statistical considerations}

The aim of this randomized phase II study was to assess the benefit of gefitinib intercalation with PC chemotherapy. The primary endpoint was objective response rate (ORR); complete response, $(\mathrm{CR})$ or partial response, (PR). When the usual ORR in the PC chemotherapy arm was $40 \%$ and an absolute increase of $15 \%$ in the ORR was obtained by intercalation of gefitinib to standard PC chemotherapy in each arm of 37 patients, the probability of correctly selecting PC chemotherapy with gefitinib as superior was 0.9. Considering follow-up loss, 42 patients were planned to be enrolled in each arm [17].

ORR was analyzed using the $\chi^{2}$ test and data were expressed with $95 \%$ confidence interval (CI). The secondary endpoints were progression-free survival (PFS) and overall survival (OS), which were assessed using the
Kaplan-Meier method with hazard ratio (HR) and $95 \%$ CI via Cox's proportional hazard model.

\section{Results}

Between April 2010 and December 2011, 90 patients were enrolled into the study and randomly assigned to receive PC with gefitinib $(N=44)$ or PC alone $(N=46)$. Finally, 43 patients in the PCG arm and 43 patients in the $\mathrm{PC}$ arm received at least one cycle of treatment (Fig. 1). Baseline characteristics were well balanced across the treatment arms (Table 1). Median age was 60 (range, 44-72) years in the PCG arm and 59 (range, 37-70) years in the PC arm. Approximately $10 \%$ of patients were non-smokers and $63 \%$ had adenocarcinoma. Patients with stage IV cancer comprised about $80 \%$ of each group equally.

\section{Primary efficacy measures}

No significant difference in ORR was observed between the two arms $(P=0.826)$, with an ORR of $41.9 \%(95 \%$ CI: $27.0-57.9$ \%) for the PCG arm and $39.5 \%$ (95 \% CI: 25.0-55.6\%) for the PC arm (Table 2). No difference in disease control rate (DCR) was observed between the two arms, with DCR values of $74.4 \%$ (95 \% CI: $58.8-$ $86.5 \%)$ and $65.1 \%$ (95 \% CI: 49.1-79.0 \%), respectively $(P=0.348)$ (Table 2).

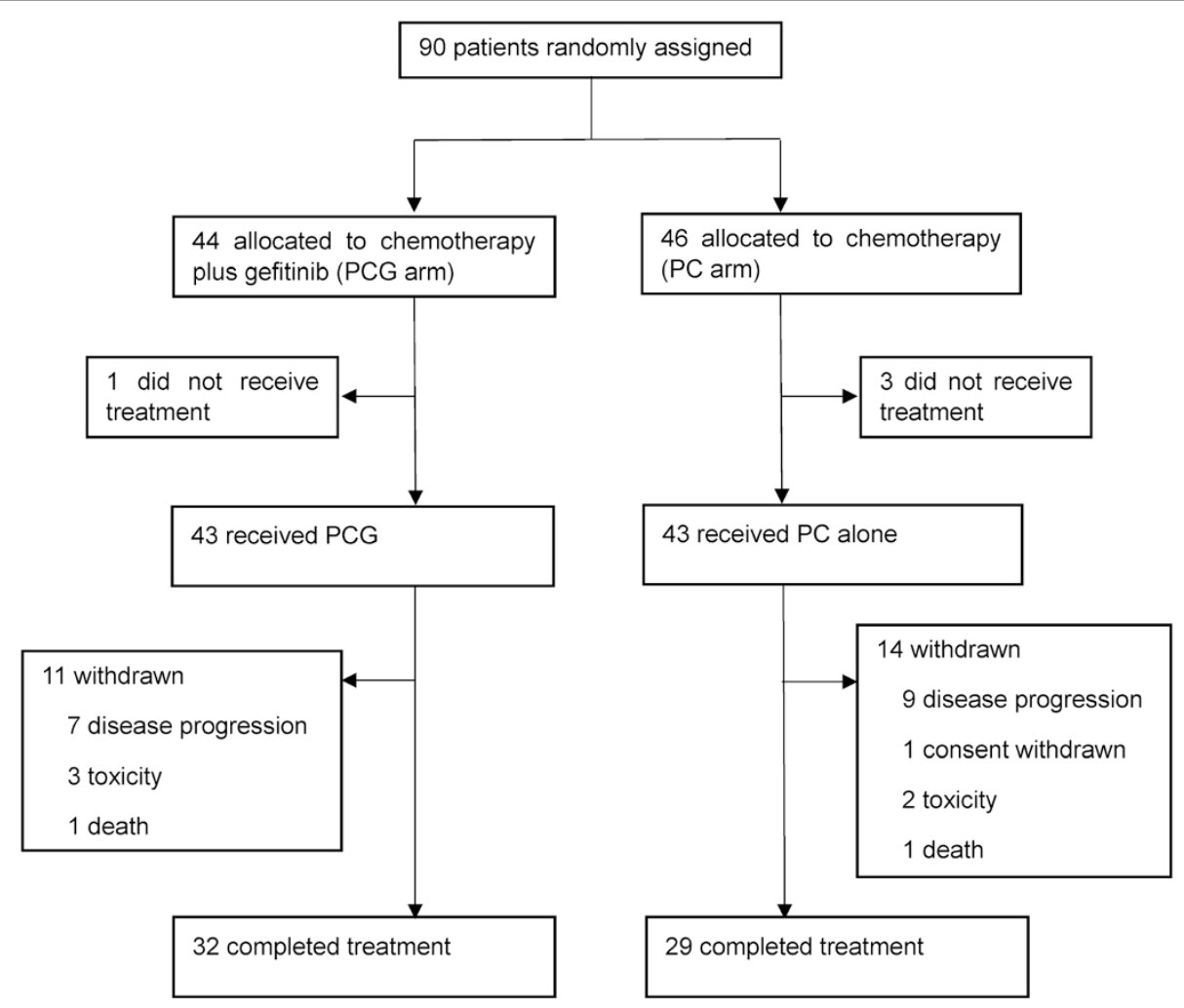

Fig. 1 Trial profile 
Table 1 Baseline characteristics of patients (intention to treat population)

\begin{tabular}{|c|c|c|c|c|}
\hline Parameter & Chemotherapy-gefitinib (PCG) $(N=44)(\%)$ & Chemotherapy (PC) $(N=46)(\%)$ & Total $(N=90)(\%)$ & $P$-value \\
\hline \multicolumn{5}{|l|}{ Age (years) } \\
\hline Median (range) & $60.0(44-72)$ & $59.0(37-70)$ & $59.5(37-72)$ & 0.678 \\
\hline \multicolumn{5}{|l|}{ Gender } \\
\hline Male & $35(79.5)$ & $42(91.3)$ & 77 (85.6) & \\
\hline Female & $9(20.5)$ & $4(8.7)$ & $13(14.4)$ & 0.113 \\
\hline \multicolumn{5}{|l|}{ Smoking status } \\
\hline Smoker & $37(84.1)$ & $44(95.7)$ & $81(90.0)$ & \\
\hline Non-smoker & $7(15.9)$ & $2(4.3)$ & $9(10.0)$ & 0.087 \\
\hline \multicolumn{5}{|l|}{ ECOG status } \\
\hline ECOG 0 & 0 & 0 & 0 & \\
\hline ECOG 1 & $44(100)$ & $46(100)$ & $90(100)$ & - \\
\hline \multicolumn{5}{|l|}{ Histological subtype } \\
\hline Adenocarcinoma & $24(54.5)$ & $31(67.4)$ & $55(61.1)$ & \\
\hline Non-adenocarcinoma & $20(45.5)$ & $15(32.6)$ & 35 (38.9) & 0.211 \\
\hline \multicolumn{5}{|l|}{ EGFR mutation } \\
\hline Wild-type & $6(13.6)$ & $3(6.5)$ & $9(10.0)$ & \\
\hline Unknown & 38 (86.4) & $43(93.5)$ & $81(90.0)$ & 0.157 \\
\hline \multicolumn{5}{|l|}{ Stage of disease } \\
\hline Stage IIIB & $8(18.2)$ & $10(21.7)$ & $18(20.0)$ & \\
\hline Stage IV & 36 (81.8) & $36(78.3)$ & $72(80.0)$ & 0.673 \\
\hline
\end{tabular}

\section{Secondary efficacy measures}

During a median follow-up of 21.7 months, there were 83 patients with PFS events (disease progression or death from any cause). No statistically significant difference in PFS was found between the two arms $(\mathrm{HR}=0.94$ [95 \% CI: 0.61-1.45], $P=0.781$ ). Median PFS was 4.1 (95 \% CI: 3.9-4.3) months in the PCG arm and 4.1 (95 \% CI: 3.9-4.3) months in the PC arm (Fig. 2).

A total of 66 patients had an OS event (death). No statistically significant difference in OS was observed between the groups ( $\mathrm{HR}=0.95$ [95 \% CI: 0.58-1.54],
$P=0.827$ ). Median OS was 9.3 (95 \% CI: 7.0-11.6) months in the PCG arm and 10.5 (95\% CI: 8.3-12.7) months in the PC arm (Fig. 3).

\section{Exploratory analyses}

Exploratory subgroup analyses are shown in Fig. 4. The negative result for the comparison between the PCG arm and the PC arm was generally consistent throughout all clinical subsets, although the small number of patients in several subsets resulted in large CIs and made the results difficult to interpret.

Table 2 Best overall response according to RECIST

\begin{tabular}{llll}
\hline Parameter & PCG arm (\%) $(N=43)$ & $P C$ arm $(\%)(N=43)$ & Odds ratio $(95 \% \mathrm{Cl})$ \\
\hline Objective response & $18(41.9)$ & $17(39.5)$ & 0.91 \\
$95 \% \mathrm{Cl}$ & $(27.0-57.9)$ & $(25.0-55.6)$ & $(0.38-2.13)$ \\
Disease control & $32(74.4)$ & $28(65.1)$ & 0.64 \\
$95 \% \mathrm{Cl}$ & $(58.8-86.5)$ & $(49.1-79.0)$ & $(0.25-1.61)$ \\
Complete response & $0(0)$ & $0(0)$ & \\
Partial response & $18(41.9)$ & $17(39.5)$ & \\
Stable disease & $14(32.6)$ & $11(25.6)$ & \\
Progressive disease & $10(23.3)$ & $13(30.2)$ & \\
Missing & $1(2.3)$ & $2(4.7)$ & \\
\hline
\end{tabular}

$N$ number; $\mathrm{Cl}$ confidence interval 


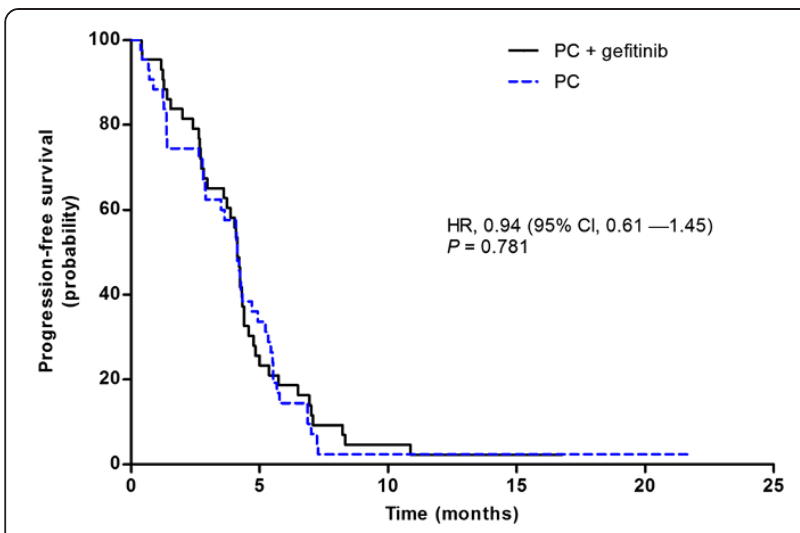

Fig. 2 Kaplan-Meier graph of progression-free survival by treatment group (ITT population)

\section{Safety}

Of the 90 patients, 86 received at least one dose of treatment and were included in the safety analyses. The median number of cycles received in both arms was 4.0.

The two arms showed a similar incidence of drugrelated toxicity. Most AEs were clinically manageable. The most commonly reported AEs of any grade were anemia, neutropenia, rash, pruritus, myalgia, neuropathy, anorexia, and cough (Table 3). Skin rash and pruritus were more common in the PCG arm (63 and $37 \%$ ) than in the PC arm (5 and $9 \%$ ), although no grade 3 rash or pruritus was observed in the PCG arm (Table 3). Frequency of diarrhea was similar in both treatment groups (Table 3). Twenty-two patients had at least one serious adverse event with CTCAE grade 3/4 [10 in the PCG arm (23.3\%) and 12 in the PC arm $(27.9 \%)$ ] (Table 3). Three patients died during the study period, two in the PCG arm (both from infection) and one in the PC arm (from pulmonary thromboembolism).

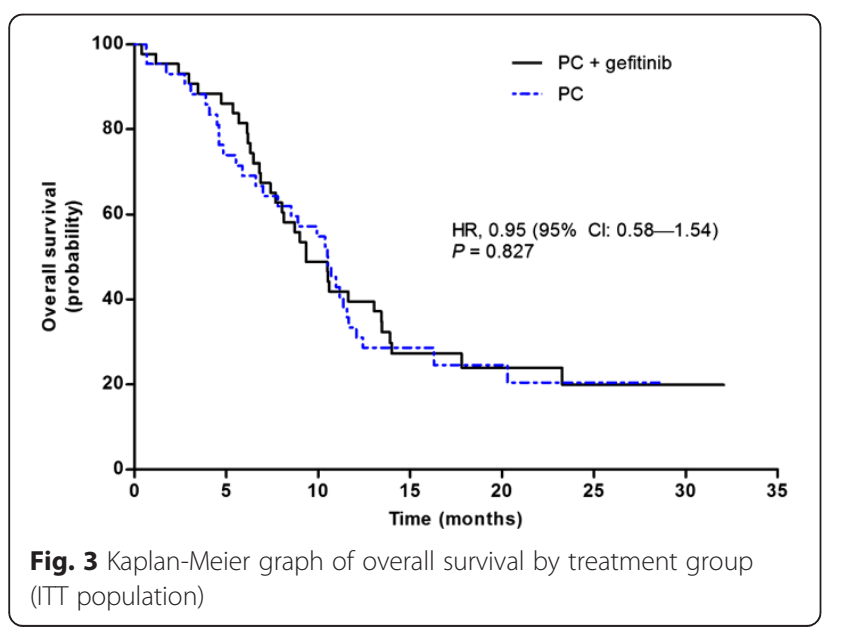

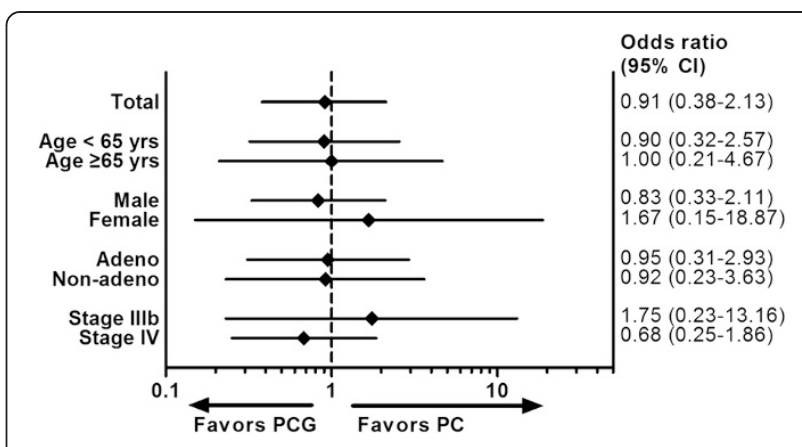

Fig. 4 Forest plots by clinical subgroups. yrs, years; adeno, adenocarcinoma

\section{Discussion}

This randomized phase II study was designed to evaluate the effect of intercalation therapy with gefitinib and paclitaxel/carboplatin chemotherapy as first-line treatment in a clinically selected population, excluding non-smoking patients with adenocarcinoma or patients with wild-type EGFR. Our study demonstrated that gefitinib intercalation did not improve the efficacy of paclitaxel/carboplatin chemotherapy in relation to ORR, PFS, and OS. Toxicity profiles were generally clinically tolerable. Combination treatment resulted in more frequent skin toxicity.

Earlier studies that assessed the combination of chemotherapy and EGFR TKIs failed to show a survival advantage. In two randomized studies, the addition of daily gefitinib or erlotinib to standard chemotherapy did not improve OS, time to progression or ORR compared with chemotherapy alone [9-11].

Two possible combination approaches have been proposed to solve this problem: a pure sequential strategy, in which chemotherapy is followed by maintenance EGFR TKI treatment $[18,19]$, and an intercalated administration strategy based on cell cycle-dependent cytotoxicity, which was supported by the results of preclinical and preliminary clinical studies [12-16].

One preclinical study assessed the effects of sequential administration of pemetrexed and erlotinib, and showed cytotoxic synergism in both mutant and wild-type EGFR cell lines [12]. In another preclinical study, the sequence-dependent synergism between paclitaxel and gefitinib was demonstrated in human lung cancer cell lines with both wild-type and mutant EGFR genes [13]. Several later phase I/II clinical studies showed that an intercalated regimen of chemotherapy and EGFR TKI is safe and effective [14-16, 20].

Recently, two clinical studies reported that the intercalated regimen offered superior efficacy compared to chemotherapy or EGFR TKIs alone [21, 22]. In the Firstline Asian Sequential Tarceva and Chemotherapy Trial (FASTACT)-2, intercalated therapy with gemcitabine plus platinum and erlotinib improved OS and PFS 
Table 3 Summary of the most common adverse events

\begin{tabular}{|c|c|c|c|c|c|c|c|c|c|}
\hline & \multicolumn{4}{|c|}{ PCG arm $(N=43)(\%)$} & \multicolumn{4}{|c|}{ PC arm $(N=43)(\%)$} & \multirow{2}{*}{$\begin{array}{l}P \text {-value } \\
\text { for all grade } A\end{array}$} \\
\hline & All grade & Gr 3 & Gr 4 & Gr 5 & All grade & Gr 3 & Gr 4 & Gr 5 & \\
\hline Patients with $\geq 1$ AE (Gr3/4/5) & $10(23)$ & & & & $12(28)$ & & & & 0.244 \\
\hline${ }^{\text {a Rash }}$ & $27(63)$ & & & & $2(5)$ & & & & $<0.001$ \\
\hline${ }^{\text {a }}$ Pruritis & $13(37)$ & & & & $4(9)$ & & & & 0.012 \\
\hline Myalgia & $25(58)$ & & & & $28(65)$ & & & & 0.506 \\
\hline Neuropathy & $21(49)$ & & & & $25(58)$ & & & & 0.387 \\
\hline Alopecia & $24(56)$ & & & & $21(49)$ & & & & 0.517 \\
\hline Anorexia & $15(35)$ & & & & $18(42)$ & & & & 0.506 \\
\hline Cough & $16(37)$ & & & & $11(26)$ & & & & 0.245 \\
\hline Nausea & $13(30)$ & & & & $8(19)$ & & & & 0.209 \\
\hline Fatigue & $10(23)$ & & & & $10(23)$ & & & & 1.000 \\
\hline Dyspepsia & $6(14)$ & & & & $7(16)$ & & & & 0.763 \\
\hline Constipation & $6(14)$ & & & & $6(14)$ & & & & 1.000 \\
\hline Diarrhea & $5(12)$ & & & & $5(12)$ & $1(2)$ & & & 1.000 \\
\hline Chest pain & $5(12)$ & & & & $5(12)$ & & & & 1.000 \\
\hline General weakness & $5(12)$ & $1(2)$ & & & $5(12)$ & & & & 1.000 \\
\hline Infection & $6(14)$ & $4(9)$ & & $2(5)$ & $3(7)$ & $3(7)$ & & & 0.483 \\
\hline TE event & $1(2)$ & & & & $2(5)$ & & $1(2)$ & $1(2)$ & 0.571 \\
\hline Neutropenia & $10(23)$ & & $2(5)$ & & $7(16)$ & $3(7)$ & $1(2)$ & & 0.660 \\
\hline Febrile neutropenia & 0 & & & & $1(2)$ & & & & 1.000 \\
\hline Anemia & $35(81)$ & $1(2)$ & & & $34(79)$ & $1(2)$ & & & 0.787 \\
\hline Thrombocytopenia & $9(21)$ & & & & $8(19)$ & $1(2)$ & & & 0.787 \\
\hline Leucopenia & $6(14)$ & $2(5)$ & & & $6(14)$ & & $1(2)$ & & 1.000 \\
\hline Increased LFT & $15(35)$ & $1(2)$ & & & $11(26)$ & & & & 0.348 \\
\hline
\end{tabular}

$A E$ adverse event; $G r$ grade; $N$ number, $L F T$ liver function test

${ }^{a}$ significant difference between two groups

compared to chemotherapy alone for unselected patients with advanced stage NSCLC as first-line setting. In subset analyses, patients with wild-type EGFR did not benefit from this intercalated regimen. [21] In a three-arm phase II study, pemetrexed-erlotinib improved PFS compared to either drug alone in a clinically selected population of never-smoking patients with non-squamous NSCLC as second-line therapy [22].

Because the combination of chemotherapy and EGFR TKIs showed cytotoxic synergism against wild-type EGFR NSCLC cell lines in a preclinical study $[12,13]$ and this combination was suggested as a new treatment option for patients with unknown EGFR status in a previous clinical study [21], we hypothesized that the intercalated strategy could be effective in patients with wildtype or unknown EGFR status. Despite the results of preclinical and clinical studies, our study failed to show the efficacy of intercalated therapy in patients with wildtype EGFR or in a clinically selected population that excluded non-smoking patients with adenocarcinoma. Although molecular tests are used routinely in clinical practice, EGFR status remains unknown in certain patients. The negative result of the present study was consistent with the results of Matjaz Zwitter et al.'s study, which showed that intercalated treatment was not of benefit for EGFR wild-type NSCLC [23].

On the other hand, intercalated treatment might be a promising approach for patients with NSCLC with EGFR mutant disease or selected patient with unknown EGFR mutation status, according to several clinical studies [21-23]. There were some explanations for the high efficacy of the intercalated therapy, including synergism of different categories of drugs and preventing repopulation of the tumor. However, a randomized trial comparing intercalated therapy with sequential treatment is needed to confirm the real value of intercalated therapy for EGFR mutated NSCLC.

\section{Conclusions}

In conclusion, the results of the present study indicated that intercalated treatment with chemotherapy and EGFR TKIs does not improve ORR, PFS, and OS compared to 
chemotherapy alone in patients in a clinically selected population excluding patients with non-smoking adenocarcinoma or mutated EGFR.

\section{Abbreviations}

EGFR: Epidermal growth factor receptor; TKI: Tyrosine kinase inhibitor; NSCLC: Non-small cell lung cancer; PCG: Paclitaxel/carboplatin with gefitinib; PC: Paclitaxel/carboplatin; ORR: Objective response rate; PFS: Progression-free survival; OS: Overall survival; Cl: Confidence interval; ECOG: Eastern Cooperative Oncology Group; PS: Performance status; AE: Adverse event; ITT: Intention-to-treat.

\section{Competing interests}

The authors have declared no competing interests.

\section{Authors' contributions}

YJC performed the data analysis and the statistical analysis, drafted the manuscript. DHL participated in the data acquisition and helped to draft the manuscript. CMC and JSL participated in the data acquisition. SJL carried out the quality control of data and algorithms. JHA participated in the design of the study. SWK conceived of the study and participated in its design and coordination and reviewed the manuscript. All authors read and approved the final manuscript.

\section{Acknowledgements}

This work was supported by the Asan Institute for Life Science [2010-430] We thank the patients and their families who took part in this study. We also thank AstraZeneca for kindly providing gefitinib.

\section{Author details}

'Department of Oncology, Asan Medical Center, University of Ulsan College of Medicine, 88 Olympic-ro-43-gil, Songpa-gu, Seoul 138-736, Korea. ${ }^{2}$ Division of Hemato-oncology, Department of Internal Medicine, Korea University Anam Hospital, Seoul, Korea. ${ }^{3}$ Asan Institute for Life Science, Asan Medical Center, Institute for Innovative Cancer Research, Seoul, Korea. ${ }^{4}$ University of Ulsan College of Medicine, Ulsan, Korea. ${ }^{5}$ Department of Oncology, Asan Medical Center, University of Ulsan College of Medicine, 88 Olympic-ro 43-gil, Songpa-gu, Seoul 138-736, Korea.

Received: 22 January 2015 Accepted: 8 October 2015

Published online: 22 October 2015

\section{References}

1. Han JY, Park K, Kim SW, Lee DH, Kim HY, Kim HT, et al. First-SIGNAL: first-line single-agent iressa versus gemcitabine and cisplatin trial in never-smokers with adenocarcinoma of the lung. J Clin Oncol. 2012:30:1122-8.

2. Maemondo M, Inoue A, Kobayashi K, Sugawara S, Oizumi S, Isobe H, et al. Gefitinib or chemotherapy for non-small-cell lung cancer with mutated EGFR. N Engl J Med. 2010;362:2380-8.

3. Mitsudomi T, Morita S, Yatabe Y, Negoro S, Okamoto I, Tsurutani J, et al. Gefitinib versus cisplatin plus docetaxel in patients with non-small-cell lung cancer harbouring mutations of the epidermal growth factor receptor (WJTOG3405): an open label, randomised phase 3 trial. Lancet Oncol. 2010;11:121-8

4. Mok TS, Wu YL, Thongprasert S, Yang CH, Chu DT, Saijo N, et al. Gefitinib or carboplatin-paclitaxel in pulmonary adenocarcinoma. N Engl J Med. 2009:361:947-57.

5. Rosell R, Carcereny E, Gervais R, Vergnenegre A, Massuti B, Felip E, et al. Erlotinib versus standard chemotherapy as first-line treatment for European patients with advanced EGFR mutation-positive non-small-cell lung cancer (EURTAC): a multicentre, open-label, randomised phase 3 trial. Lancet Oncol. 2012;13:239-46.

6. Zhou C, Wu YL, Chen G, Feng J, Liu XQ, Wang C, et al. Erlotinib versus chemotherapy as first-line treatment for patients with advanced EGFR mutation-positive non-small-cell lung cancer (OPTIMAL, CTONG-0802): a multicentre, open-label, randomised, phase 3 study. Lancet Oncol. 2011;12:735-42.

7. Lynch TJ, Bell DW, Sordella R, Gurubhagavatula S, Okimoto RA, Brannigan BW, et al. Activating mutations in the epidermal growth factor receptor underlying responsiveness of non-small-cell lung cancer to gefitinib. N Engl J Med. 2004:350:2129-39.
8. Paez JG, Janne PA, Lee JC, Tracy S, Greulich H, Gabriel S, et al. EGFR mutations in lung cancer: correlation with clinical response to gefitinib therapy. Science. 2004;304:1497-500

9. Giaccone G, Herbst RS, Manegold C, Scagliotti G, Rosell R, Miller V, et al. Gefitinib in combination with gemcitabine and cisplatin in advanced nonsmall-cell lung cancer: a phase III trial-INTACT 1. J Clin Oncol. 2004;22:777-84.

10. Herbst RS, Giaccone G, Schiller JH, Natale RB, Miller V, Manegold C, et al. Gefitinib in combination with paclitaxel and carboplatin in advanced nonsmall-cell lung cancer: a phase III trial-INTACT 2. J Clin Oncol. 2004;22:785-94.

11. Herbst RS, Prager D, Hermann R, Fehrenbacher L, Johnson BE, Sandler A, et al. TRIBUTE: a phase III trial of erlotinib hydrochloride (OSI-774) combined with carboplatin and paclitaxel chemotherapy in advanced non-small-cell lung cancer. J Clin Oncol. 2005;23:5892-9.

12. Li T, Ling YH, Goldman ID, Perez-Soler R. Schedule-dependent cytotoxic synergism of pemetrexed and erlotinib in human non-small cell lung cancer cells. Clin Cancer Res. 2007;13:3413-22.

13. Cheng $\mathrm{H}$, An SJ, Zhang XC, Dong S, Zhang YF, Chen ZH, et al. In vitro sequence-dependent synergism between paclitaxel and gefitinib in human lung cancer cell lines. Cancer Chemother Pharmacol. 2011;67:637-46.

14. Davies AM, Ho C, Beckett L, Lau D, Scudder SA, Lara PN, et al. Intermittent erlotinib in combination with pemetrexed: phase I schedules designed to achieve pharmacodynamic separation. J Thorac Oncol. 2009:4:862-8.

15. Ranson M, Reck M, Anthoney A, Hanauske AR, Dean E, Melezinek I, et al. Erlotinib in combination with pemetrexed for patients with advanced nonsmall-cell lung cancer (NSCLC): a phase I dose-finding study. Ann Oncol. 2010;21:2233-9.

16. Mok TS, Wu YL, Yu CJ, Zhou C, Chen YM, Zhang L, et al. Randomized, placebo-controlled, phase II study of sequential erlotinib and chemotherapy as first-line treatment for advanced non-small-cell lung cancer. J Clin Oncol. 2009;27:5080-7.

17. Simon R, Wittes RE, Ellenberg SS. Randomized phase II clinical trials. Cancer Treat Rep. 1985;69:1375-81

18. Cappuzzo F, Ciuleanu T, Stelmakh L, Cicenas S, Szczesna A, Juhasz E, et al. Erlotinib as maintenance treatment in advanced non-small-cell lung cancer: a multicentre, randomised, placebo-controlled phase 3 study. Lancet Oncol. 2010;11:521-9.

19. Zhang L, Ma S, Song X, Han B, Cheng Y, Huang C, et al. Gefitinib versus placebo as maintenance therapy in patients with locally advanced or metastatic non-small-cell lung cancer (INFORM; C-TONG 0804): a multicentre, double-blind randomised phase 3 trial. Lancet Oncol. 2012;13:466-75.

20. Zwitter M, Rajer M, Kovac V, Kern I, Vrankar M, Smrdel U. Intermittent chemotherapy and erlotinib for nonsmokers or light smokers with advanced adenocarcinoma of the lung: a phase II clinical trial. J Biomed Biotechnol. 2011;2011:185646

21. Wu YL, Lee JS, Thongprasert S, Yu CJ, Zhang L, Ladrera G, et al. Intercalated combination of chemotherapy and erlotinib for patients with advanced stage non-small-cell lung cancer (FASTACT-2): a randomised, double-blind trial. Lancet Oncol. 2013;14:777-86.

22. Lee DH, Lee JS, Kim SW, Rodrigues-Pereira J, Han B, Song XQ, et al. Threearm randomised controlled phase 2 study comparing pemetrexed and erlotinib to either pemetrexed or erlotinib alone as second-line treatment for never-smokers with non-squamous non-small cell lung cancer. Eur J Cancer. 2013;49:3111-21

23. Zwitter M, Stanic K, Rajer M, Kern I, Vrankar M, Edelbaher N, et al. Intercalated chemotherapy and erlotinib for advanced NSCLC: high proportion of complete remissions and prolonged progression-free survival among patients with EGFR activating mutations. Radiol Oncol. 2014;48:361-8. 